\title{
Fragmentation Pathways of $\mathrm{N}^{\mathrm{G}}$-Methylated and Unmodified Arginine Residues in Peptides Studied by ESI-MS/MS and MALDI-MS
}

\author{
Peter M. Gehrig* and Peter E. Hunziker \\ Institute of Biochemistry, University of Zurich, Zurich, Switzerland \\ Sotir Zahariev and Sándor Pongor \\ International Center for Genetic Engineering and Biotechnology (ICGEB), Trieste, Italy
}

\begin{abstract}
Protein methylation at arginine residues is a prevalent posttranslational modification in eukaryotic cells that has been implicated in processes from RNA-binding and transporting to protein sorting and transcription activation. Three main forms of methylarginine have been identified: $\mathrm{N}^{\mathrm{G}}$-monomethylarginine (MMA), asymmetric $\mathrm{N}^{\mathrm{G}}, \mathrm{N}^{\mathrm{G}}$-dimethylarginine (aDMA), and symmetric $\mathrm{N}^{\mathrm{G}}, \mathrm{N}^{\prime} \mathrm{G}$-dimethylarginine (sDMA). To investigate gas-phase fragmentations and characteristic ions arising from methylated and unmodified arginine residues in detail, we subjected peptides containing these residues to electrospray triple-quadrupole tandem mass spectrometry. A variety of low mass ions including (methylated) ammonium, carbodiimidium, and guanidinium ions were observed. Fragment ions resulting from the loss of the corresponding neutral fragments (amines, carbodiimide, and guanidine) from intact molecular ions as well as from $\mathrm{N}$ - and $\mathrm{C}$-terminal fragment ions were also identified. Furthermore, the peptides containing either methylated or unmodified arginines gave rise to abundant fragment ions at $\mathrm{m} / \mathrm{z} 70,112$, and 115 , for which cyclic ion structures are proposed. Electrospray ionization tandem mass spectra revealed that dimethylammonium $(\mathrm{m} / \mathrm{z} 46)$ is a specific marker ion for aDMA. A precursor ion scanning method utilizing this fragment ion was developed, which allowed sensitive and specific detection of aDMA-containing peptides even in the presence of a five-fold excess of phosphorylase B digest. Interestingly, regular matrix-assisted laser desorption/ionization mass spectra recorded from aDMA- or sDMA-containing peptides showed metastable fragment ions resulting from cleavages of the arginine side chains. The neutral losses of mono- and dimethylamines permit the differentiation between aDMA and sDMA. (J Am Soc Mass Spectrom 2004, 15, 142-149) (C) 2004 American Society for Mass Spectrometry
\end{abstract}

$\mathrm{P}$ osttranslational modifications of proteins allow the cell to expand its repertoire beyond the constraints imposed by the twenty encoded amino acids. Because many biological processes are controlled by such modifications, their analysis is an essential step in the investigation of biological pathways. Arginine methylation in proteins was discovered over 35 years ago [1]. The possible functional roles of this modification and the characterization of the enzymes involved have been examined in recent reviews [2, 3]. Two principal categories of protein arginine methyltrans-

Published online December 16, 2003

Address reprint requests to Dr. P. M. Gehrig, Functional Genomics Center Zurich, Winterthurerstrasse 190, 8057 Zurich, Switzerland. E-mail: peter.gehrig@fgcz.unizh.ch

${ }^{*}$ Current address: Functional Genomics Center Zurich, Winterthurerstrasse 190, 8057 Zurich, Switzerland ferases (PRMTs) transfer methyl groups from S-adenosyl-L-methionine to the guanidino group of arginine residues. Both types of enzymes catalyze the formation of $\mathrm{N}^{\mathrm{G}}$-monomethylarginine (MMA) in proteins. Type I PRMT enzymes also produce asymmetric $\mathrm{N}^{\mathrm{G}}, \mathrm{N}^{\mathrm{G}}$-dimethylarginine (aDMA), whereas Type II enzymes form symmetric $\mathrm{N}^{\mathrm{G}}, \mathrm{N}^{\prime} \mathrm{G}$-dimethylarginine (sDMA). Type I substrates include many RNA binding and transporting proteins, transcription factors, nuclear matrix proteins and cytokines. Arginine methylation by Type I enzymes usually occurs in conserved glycine- and arginine-rich (GAR) sequences, typically in RG or RGG sequences [2, 4]. Myelin basic protein and four spliceosomal proteins are the only substrates for Type II arginine methyltransferases reported in the literature $[5,6]$. Intriguingly, the context of methylated residues in these proteins (GRG) differs from the consensus sequence for asymmetrically dimethylated proteins. Most PRMT genes discovered to 
date encode for Type I enzymes [3, 7], but recent data have revealed that PRMT5/JBP1 (Janus kinase binding protein 1) is a Type II methyltransferase [8]. A novel modification of arginine residues, $\delta$-N-methylarginine, discovered in yeast proteins, indicates that an additional type of protein arginine methyltransferase exists in nature [9].

Widely-used methods for detecting and analyzing the modified residues include the incorporation of radioactive methyl $-{ }^{3} \mathrm{H}$ groups, amino acid analyses, Edman sequencing and peptide mass mapping by matrix-assisted laser desorption/ionization (MALDI) mass spectrometry. The Edman sequencing technique and MALDI mass spectrometry have been utilized for the location of arginine methylation sites. Amino acid analyses and Edman sequencing allow the differentiation between asymmetrically and symmetrically dimethylated arginine residues in principle. In general, trypsin is the enzyme of choice for generating peptide maps of known proteins, as well as for proteomics applications. In trypsin digests of proteins containing either aDMA and MMA [4] or sDMA and MMA [5], no peptides resulting from cleavages after modified arginines were identified. Synthetic peptides containing up to eight aDMA residues were 4 to 20 times more resistant to trypsin proteolysis than their non-methylated counterparts [10].

In the study reported here, fragmentation pathways of peptides containing $\mathrm{N}^{\mathrm{G}}$-methylated or unmodified arginine residues were investigated using an electrospray ionization (ESI) triple-quadrupole mass spectrometer. The sequences of the testpeptides correspond to arginine methylation sites of nucleolin, an abundant aDMA-containing protein of the nucleolus [11], and of myelin basic protein, which carries a single monomethylated or symmetrically dimethylated arginine residue [5]. A surprising variety of fragment ions arising from methylated or even from unmethylated arginines were identified. Certain fragments observed in the low energy collision-induced dissociation spectra permit the unambiguous differentiation between asymmetric and symmetric dimethylarginine residues. Precursor ion scanning is a valuable tool for the selective detection of modified peptides in complex mixtures [12, 13]. We developed a precursor ion experiment for the sensitive and selective detection of aDMA-containing peptides. Dimethylammonium $(\mathrm{m} / \mathrm{z} 46)$, which was found to be formed exclusively from this modified amino acid residue, was utilized as "reporter" ion. During final preparation of this manuscript, a report by Rappsilber et al. appeared that also describes a precursor ion scanning method for the analysis of arginine dimethylated peptides [14]. Interestingly, some of the fragment ions observed in the ESI collision-induced dissociation (CID) spectra appeared as metastable ions in regular MALDI mass spectra and also permitted the distinction between the two isomeric forms of DMA.

\section{Experimental}

\section{Materials}

Peptides GXGGFGGXGGFXGGXGG-NH ${ }_{2}$ (X: arginine or aDMA) were synthesized by a solid phase method using Fmoc chemistry. Fmoc-aDMA(Mts)-OH was synthesized as described [10]. A $30 \mu \mathrm{M}$ solution of these peptides in $20 \mathrm{mM}$ ammonium bicarbonate buffer, $\mathrm{pH}$ 8.0, was cleaved with $1.5 \%(\mathrm{w} / \mathrm{w})$ chymotrypsin (Roche Molecular Biochemicals, Basle, Switzerland) for $1 \mathrm{~h}$ at $25^{\circ} \mathrm{C}$. The chymotryptic cleavage primarily generated the following fragments: GXGGFGGXGGF and XGGXGG-NH ${ }_{2}$. Glycogen phosphorylase B from rabbit (Sigma, St. Louis, MO, $100 \mu \mathrm{M}$ ) was digested with $1 \%$ $(\mathrm{w} / \mathrm{w})$ of modified trypsin (Promega, Madison, WI) in $1 \mathrm{mM}$ Tris buffer, $\mathrm{pH} 8.5$, at $37^{\circ} \mathrm{C}$ over night. The digestion of myelin basic protein from bovine brain (Sigma; $50 \mu \mathrm{M}$ ) with modified trypsin was performed in $100 \mathrm{mM}$ ammonium bicarbonate buffer, $\mathrm{pH}$ 8.0, and $2 \mathrm{mM} \mathrm{CaCl}_{2}$, at $37^{\circ} \mathrm{C}$ for $6 \mathrm{~h}$. The MMA- and sDMA-containing peptides of interest were isolated by liquid chromatography using a $C_{18}$ reversed-phase column $(1 \mathrm{~mm}$ i.d. $\times 250 \mathrm{~mm}$ length $)$ and detected on-line by ESI mass spectrometry. The concentrations of the peptides used in the precursor ion scan experiments and of glycogen phosphorylase B were determined by amino acid analyses using an AminoQuant II analyzer (Agilent Technologies, Palo Alto, CA) with fluorescence detection.

\section{Mass spectrometry}

ESI mass spectra and tandem mass spectra were recorded on an API $\mathrm{III}^{+}$triple-quadrupole instrument (AB/MDS Sciex, Concord, ON, Canada). Mass spectra and tandem mass spectra were acquired at unit mass resolution, permitting the distinction between singly and doubly charged ions. For the acquisition of tandem mass spectra, argon was used as the collision gas at a pressure of approximately $2 \times 10^{-2}$ torr. The collision energy was adjusted for each experiment individually. In the precursor ion mode, both mass-selective quadrupoles were optimized for sensitivity at the expense of some resolution. Precursor ion scans were acquired with a step size of $0.25 \mathrm{Da}$ and a dwell time of $1.4 \mathrm{~ms}$. The optimal collision gas pressure was approximately $1.2 \times 10^{-2}$ torr and the collision energy setting was $38 \mathrm{~V}$ (difference between R0 and R2 potentials on the API $\mathrm{III}^{+}$instrument).

MALDI time-of-flight (TOF) mass spectra were recorded on a Bruker (Leipzig, Germany) Biflex instrument in the reflector mode employing pulsed ion extraction. The MALDI spectra were calibrated externally using ion signals from standard peptides. $\alpha$-Cyano-4hydroxycinnamic acid (Fluka, Buchs, Switzerland) was used as the matrix. 


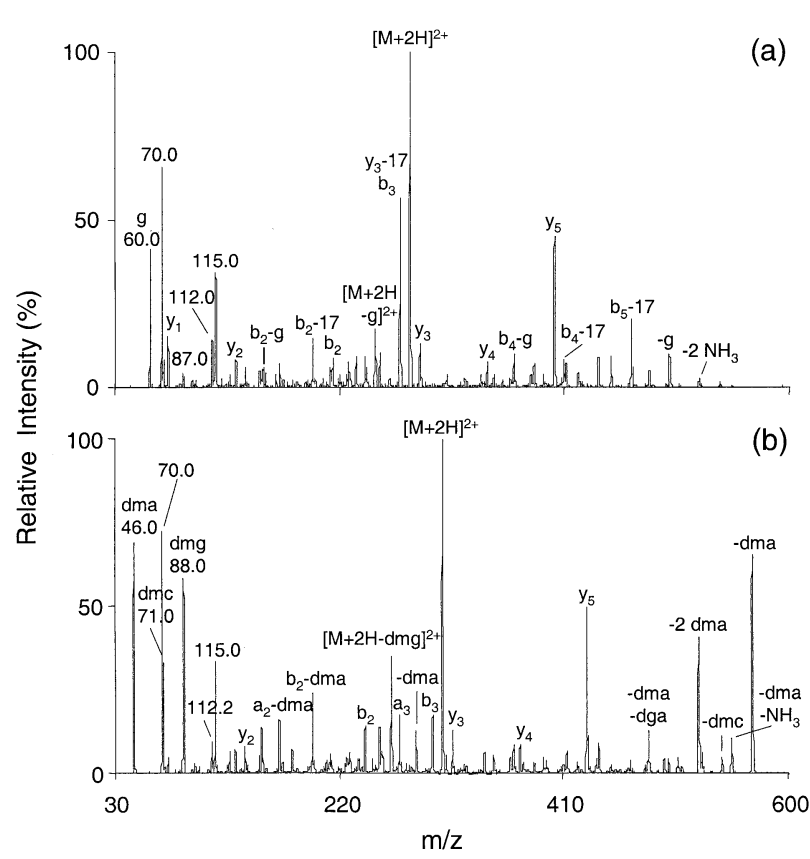

Figure 1. Tandem CID mass spectra (a) of peptide RGGRGG$\mathrm{NH}_{2}$ and (b) of peptide aDMA-GG-aDMA-GG-NH ${ }_{2}(5 \mu \mathrm{M}$ each). Abbreviations: g, guanidine; dma, dimethylamine; dmc, dimethylcarbodiimide; $\mathrm{dmg}$, dimethylguanidine. The abbreviations include the corresponding ions.

\section{Results}

In order to characterize fragment ions and neutral fragments resulting from unmodified arginine and from aDMA in detail, the nucleolin-derived peptides RGGRGG-NH $\mathrm{N}_{2}$ and aDMA-GG-aDMA-GG-NH $\mathrm{NH}_{2}$ were subjected to MS/MS analysis by ESI triple-quadrupole mass spectrometry (Figure 1). When subjected to CID, the peptides yielded surprisingly complex spectra. In addition to the peptide backbone fragmentation $b$ and $y$ ion series, a variety of fragment ions and losses of neutral fragments related to unmodified arginine or aDMA were identified (Schemes 1 and 2). Typically, these ions involve one of three cleavages of the guanidino group of the arginine residues.

Relatively intense ion signals arise from loss of guanidinium ions or of neutral guanidine from the arginine side chains (Cleavage $\mathbf{1}$ in Scheme 1). In the spectrum recorded from peptide RGGRGG-NH (Fig- $^{2}$ ure 1a), the signal at $m / z 60$ corresponds to the guanidinium ion (Structure 1 in Scheme 2), and the signal at $\mathrm{m} / \mathrm{z} 499$ is consistent with the singly charged peptide ion upon loss of guanidinium. Furthermore, formal losses of the neutral fragments ammonia and guanidine from the doubly charged precursor ion and from $b$ and $y$ ions were observed. It is not known if the losses occur from the precursor with all subsequent losses occurring from that structure or if the $\mathrm{b}$ and $\mathrm{y}$ ions directly lose the small neutrals.

Most amino acid residues give rise to immonium ions $(\mathrm{HN}=\mathrm{CH}-\mathrm{R}$; labeled in a spectrum with the single<smiles>CNC(C)C(C)=O</smiles>

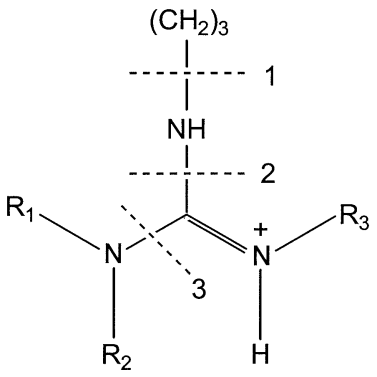

$\mathrm{R}_{1}=\mathrm{R}_{2}=\mathrm{R}_{3}=\mathrm{H}$ for Arg

$R_{1}=M e, R_{2}=R_{3}=H$ for MMA

$R_{1}=R_{2}=M e, R_{3}=H$ for aDMA

$R_{1}=H, R_{2}=R_{3}=$ Me for $s D M A$
Scheme 1. Chemical structure and side chain fragmentations of arginine and the methylated arginines studied. Cleavage 1 leads to guanidines and guanidinium ions, Cleavage 2 to neutral or protonated carbodiimides, and Cleavage 3 to amines or ammonium ions.

letter code of the particular amino acid) with a mass 27 Da lower than that of the amino acid itself [15-17]. However, the regular immonium ion derived from arginine $(m / z 129)$ is completely absent (Figure 1a). Instead, arginine residues appear to give rise to several other low mass ions, the proposed structures of which are illustrated in Scheme 2. The weak signal at $\mathrm{m} / \mathrm{z} 87$ may arise from the loss of carbodiimide from the immonium ion of arginine (Cleavage $\mathbf{2}$ in Scheme 1), which results in the immonium ion of ornithine (Structure 3 ). This ion tends to cyclize and concomitantly release ammonia, leading to the formation of the stable ion of $\mathrm{m} / \mathrm{z} 70$ (Structure 2). Alternatively, a fragment ion of $\mathrm{m} / \mathrm{z} 70$ may be generated by the loss of guanidine

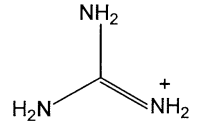

$1, \mathrm{~m} / \mathrm{z} 60.056$

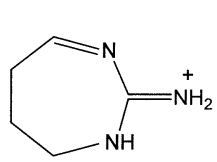

$\underline{4}, \mathrm{~m} / \mathrm{z} 112.087$

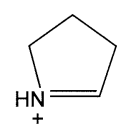

2, $\mathrm{m} / \mathrm{z} 70.066$

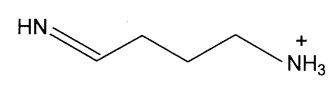

$\underline{3}, \mathrm{~m} / \mathrm{z} 87.092$

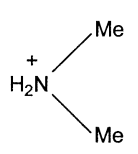

$\underline{6}, \mathrm{~m} / \mathrm{z} 46.066$

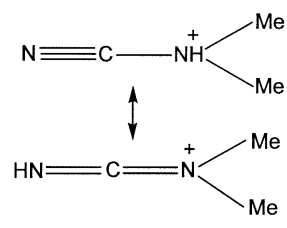

7, $\mathrm{m} / \mathrm{z} 71.061$

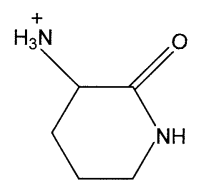

$\underline{5}, \mathrm{~m} / \mathrm{z} 115.087$
Scheme 2. Structures proposed for selected fragment ions arising from unmodified arginines or from aDMA residues in peptides. Analogous fragment ions may be generated from MMA or from sDMA residues. 
(Cleavage 1) from the immonium ion of arginine $(\mathrm{m} / \mathrm{z}$ 129). The signal at $m / z 112$ can be best explained by a cyclization of the immonium ion of arginine and accompanying loss of ammonia (Structure 4). The abundant ion at $\mathrm{m} / \mathrm{z} 115$ may be best rationalized by the loss of carbodiimide (Cleavage 2) and subsequent cyclization, eventually leading to the formation of the Cyclic Ion 5. Remarkably, intramolecular $\delta$-N-lactams are frequently observed as side products resulting from arginine activation or coupling during peptide syntheses [18].

The low-energy CID spectrum acquired from the peptide carrying two aDMA residues, aDMA-GGaDMA-GG- $\mathrm{NH}_{2}$ (Figure $1 \mathrm{~b}$ ), exhibit two intense peaks corresponding to dimethylammonium $(\mathrm{m} / \mathrm{z} 46$, Structure 6) and to the peptide ion resulting from the release of dimethylammonium $(\mathrm{m} / \mathrm{z} 569$; Cleavage 3$)$. These fragment ions and the formal loss of dimethylamine from $b$ and $y$ ions and from the precursor ion confirm the presence of the modification and allow to unambiguously distinguish between aDMA and sDMA. A peak of medium intensity at $m / z 71$ is likely to correspond to dimethylcarbodiimidium and/or cyano-dimethylammonium (Structure 7), and a somewhat more intense peak arises from dimethylated guanidinium (Structure $8, \mathrm{~m} / \mathrm{z} 88$ ). These two fragment ions are also characteristic of DMA residues, but they do not permit the differentiation of its isomeric forms. Dimethylguanidine was released primarily as a neutral fragment from the peptide ion, giving rise to the doubly charged fragment ion at $\mathrm{m} / \mathrm{z} 264$. Prominent signals at $\mathrm{m} / \mathrm{z} 70$ (Structure 2) and $m / z 115$ (Structure 5) and a minor one at $m / z 112$ (Structure 4), which are compatible with aDMA, appeared in the spectrum. Essentially the same fragmentations were observed in the CID spectra of the nucleolin-derived peptide GRGGFGGRGGF and of its aDMA-containing counterpart (data not shown).

Myelin basic protein, which was one of the first arginine-methylated proteins identified [5], stands in contrast to most methylated RNA-binding proteins in that it contains SDMA residues in addition to MMA. To investigate fragment ions arising from MMA and sDMA, CID spectra were recorded from peptides GMMA-GLSLSR and G-sDMA-GLSLSR, which were obtained by digestion of myelin basic protein with trypsin. The CID spectrum recorded from the former peptide (Figure 2a) indicates that the side chains of MMA residues are rather stable in comparison to sDMA. The signal at $m / z 828$ is due to the loss of monomethylammonium from the doubly charged peptide ion. The loss of methylguanidine and monomethylcarbodiimide from $b_{2}$ and from the peptide ion as well as the methylguanidinium ion $(\mathrm{m} / \mathrm{z} 74)$ were also observed.

The comparison of this MS/MS spectrum to that acquired from the peptide containing sDMA (Figure 2b) reveals that the second methyl group further destabilizes the arginine side chain, resulting in more abundant arginine-related fragment ions. The bond between the internal (or $\delta$ ) nitrogen atom and the carbon atom of the guanidino group of sDMA is particularly unstable

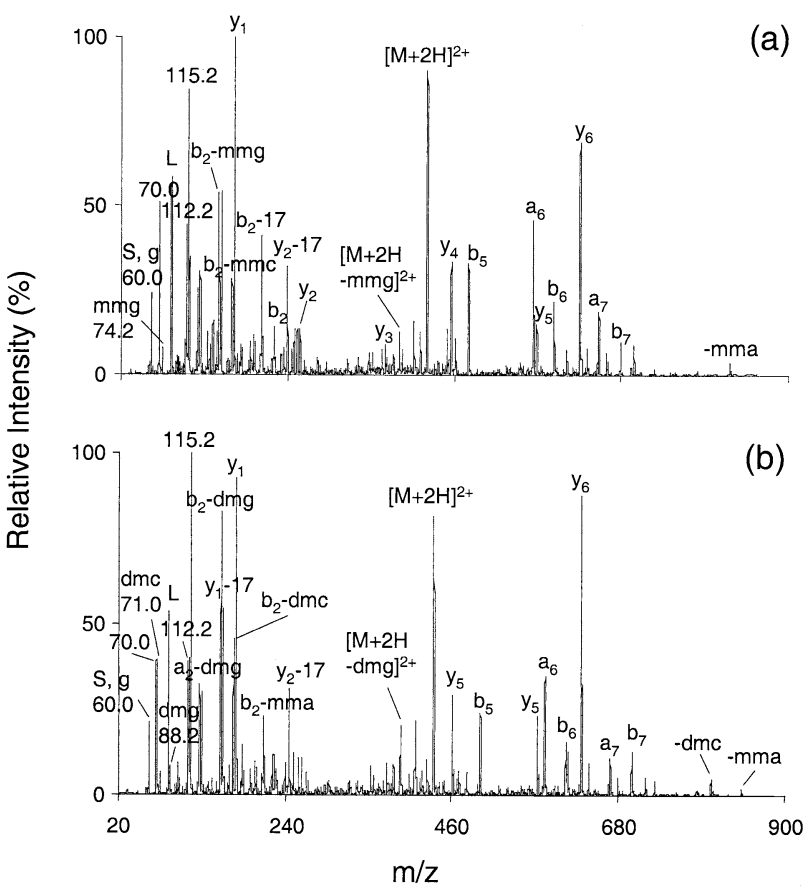

Figure 2. Tandem CID mass spectra (a) of peptide G-MMAGLSLSR and (b) of peptide G-sDMA-GLSLSR. Abbreviations: S, immonium ion of serine; L, immonium ion of leucine; g, guanidine; $\mathrm{mmg}$, monomethylguanidine; $\mathrm{mmc}$, monomethylcarbodiimide; mma, monomethylamine; dmc, dimethylcarbodiimide; dmg, dimethylguanidine. The abbreviations include the corresponding ions.

and susceptible to gas phase fragmentation (Cleavage 2). The CID spectrum recorded from peptide G-sDMAGLSLSR shows a fairly intense signal at $\mathrm{m} / \mathrm{z} 71$ corresponding to dimethylcarbodiimidium, and the signal at $\mathrm{m} / \mathrm{z} 803$ is consistent with the peptide remaining upon this cleavage. Other intense peaks can be rationalized with the formal loss of monomethylamine, dimethylcarbodiimide, or dimethylguanidine from $a_{2}$ or $b_{2}$ ions. The fragment ions of $m / z 70,112$, and 115 (Scheme 2) were observed in the CID spectra recorded from the peptides containing methylarginine or sDMA, too. However, these fragments were probably formed at least partially from the C-terminal unmodified arginine in these peptides.

The common immonium ions and immonium ion fragments have been identified and are routinely used in sequencing algorithms [15-17]. High-energy CID spectra recorded from peptides containing arginine indicated that ions of $m / z 70,87,100,112$, and 129 are diagnostically important low mass fragment ions arising from this amino acid residue. While the ions at $\mathrm{m} / \mathrm{z}$ 70,87 , and 112 were actually observed in the lowenergy CID spectrum shown in Figure 1a, the regular immonium ion of arginine $(m / z$ 129) decayed completely. The ion at $\mathrm{m} / \mathrm{z} 100$ appears to require the cleavage of a carbon-carbon bond and is probably formed only under high-energy CID conditions.

The release of guanidine from arginine side chains 
was first reported for ESI low-energy CID spectra of cyclic heptapeptide microcystins [19]. The loss of dimethylamine, guanidine, and dimethylguanidine from a linear peptide containing both arginine and aDMA was observed in a CID mass spectrum recorded by an ESI ion trap instrument [20]. The fragmentation pattern of the aDMA side chain allowed the unambiguous assignment of the isomeric form of the modified arginine residue. ESI-MS/MS spectra acquired from the free amino acid arginine and from its methylated derivatives MMA, aDMA, and sDMA indicated that amines, carbodiimides and guanidines were released from the guanidino group [21]. In a recent study conducted on a quadrupole time-of-flight instrument, DMA-containing peptides were shown to give rise to five characteristic fragments: $\mathrm{m} / \mathrm{z} 46$ and $\mathrm{m} / \mathrm{z} 71$, and neutral loss of 45 for asymmetric dimethylation and $m / z 71$ as well as neutral loss of 70 for symmetric dimethylation [14]. The corresponding signals were much weaker than those observed in this study, and other fragment ions described here were not identified. It appears that the relativ intensities of these fragments is dependent both on the amino acid sequences of the peptides and on the type of mass spectrometer utilized. Triple quadrupole and hybrid quadrupole linear ion trap mass spectrometers are particularly well suited for the detection of low mass fragment ions, while quadrupole time-of-flight instruments exhibit some bias against ions of low mass. The fragment ions described here would typically fall below the cutoff of ion trap mass spectrometers.

Precursor ion scanning methods are frequently utilized to detect posttranslational protein modifications. When performing a precursor ion scanning experiment the mass spectrometer searches for all ions that fragment to produce a common, diagnostic product ion. If these fragment ions have unique $\mathrm{m} / \mathrm{z}$ values, peptides carrying these modifications may be selectively detected by precursor ion scanning within a peptide mixture using an electrospray tandem mass spectrometer. Precursor ion scanning methods have been developed for phosphorylated amino acids in general [13, 22], for phosphotyrosines [23], for $\mathrm{N}$ - and O-linked glycopeptides [12], for O-GlcNAc-modified peptides [24], and for various less common protein modifications.

Here we report a precursor ion scanning experiment for the specific detection of peptides containing aDMA. The CID mass spectra presented in Figures 1 and 2 reveal that low mass fragment ions of $m / z 70,112$, and 115 are generated from both unmodified and methylated arginine residues. No sufficiently intense fragment ion signal that would be specific for MMA was observed in the CID mass spectrum of peptide G-MMAGLSLSR. The $\mathrm{m} / \mathrm{z}$ value of monomethylguanidinium $(\mathrm{m} / \mathrm{z} 74)$ corresponds to that of the immonium ion of threonine. The CID spectra of peptides with aDMA as well as sDMA exhibit peaks of medium intensity at $\mathrm{m} / \mathrm{z}$ 71 and 88 . However, the $m / z$ value of the dimethylguanidinium ion $(\mathrm{m} / \mathrm{z} 88)$ coincides with that of the immo-

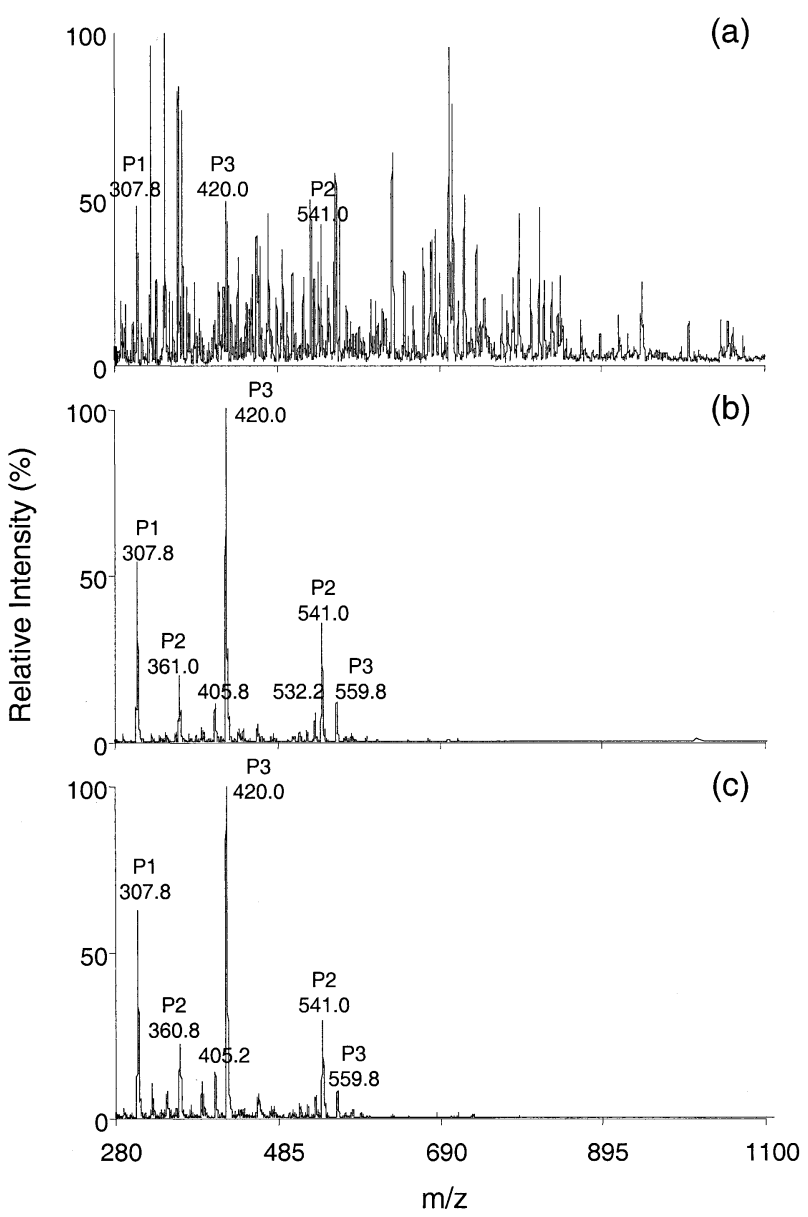

Figure 3. (a) ESI mass spectrum recorded from a 1:1 mixture (2 $\mu \mathrm{M})$ of a tryptic glycogen phosphorylase $\mathrm{B}$ digest and of test peptides aDMA-GG-aDMA-GG-NH ${ }_{2}$ (P1), G-aDMA-GGFGGaDMA-GGF (P2), and G-aDMA-GGFGG-aDMA-GGF-aDMA-GGaDMA-GG-NH $\mathrm{N}_{2}$ (P3). (b) Precursor ion scan spectrum of the same glycogen phosphorylase $\mathrm{B} /$ testpeptide mixture obtained by selecting the fragment ion at $\mathrm{m} / \mathrm{z} 46$. The minor ion signals at $\mathrm{m} / \mathrm{z} 405$ and 532 were assigned to byproducts of the peptide synthesis, which also contain aDMA. (c) Precursor ion scan spectrum recorded from a 5:1 mixture of glycogen phosphorylase B digest (2 $\mu \mathrm{M})$ and of the testpeptides $(0.4 \mu \mathrm{M})$.

nium ion of aspartic acid. The fragment ion at $m / z 71$ may be difficult to differentiate from the heavy isotope peak of Ion 2 (Scheme 2), which arises from arginine and proline residues and tends to be very abundant (Figures 1 and 2). The CID mass spectra recorded from peptides aDMA-GG-aDMA-GG-NH ${ }_{2}$ and G-aDMAGGFGG-aDMA-GGF indicate that peptides carrying aDMA give rise to a unique and intense signal at $m / z 46$. We investigated the sensitivity and specificity of precursor ion scanning experiments utilizing this ion as a characteristic marker of aDMA. Figure 3a shows a regular ESI mass spectrum recorded from a 1:1 mixture (mol:mol) of a tryptic digest of phosphorylase B and of three aDMA-containing test peptides. The spectrum obtained by precursor of $\mathrm{m} / \mathrm{z} 46$ scans is displayed in Figure $3 \mathrm{~b}$. All prominent precursor ion signals are attributable to the three aDMA-containing peptides, 
and a few minor signals correspond to related peptide byproducts. Only the three most abundant peptide ions at $m / z 308,420$, and 541 are observable in the regular mass spectrum, too. To test the applicability of this approach to the detection of incompletely dimethylated arginines, the aDMA peptides were combined with a fivefold excess of phosphorylase digest, and the mixture was subjected to precursor of $\mathrm{m} / \mathrm{z} 46$ scanning (Figure 3c). Even in this precursor ion scan spectrum, all major signals could be assigned to aDMA-containing peptides. These results imply that the precursor ion scanning experiment based on the marker ion of $\mathrm{m} / \mathrm{z} 46$ permits the sensitive and specific detection of aDMAcontaining peptides and that virtually no interfering peptide-derived fragment ions are found at this $\mathrm{m} / \mathrm{z}$ value. This conclusion is in agreement with a recently published paper of Rappsilber et al. on the detection of arginine dimethylated peptides by parallel precursor ion scanning mass spectrometry [14]. The method utilizes two side-chain fragments of DMA of $\mathrm{m} / \mathrm{z} 46$ (Structure 6, Scheme 2) and $\mathrm{m} / \mathrm{z} 71$ (Structure 7) for precursor ion scanning on a quadrupole time-of-flight mass spectrometer. The precursor of $m / z 46$ scans were found to be very specific for aDMA-containing peptides, while the $m / z 71$ scans gave rise to a few signals which could not be assigned to aDMA- or sDMAcontaining peptides.

Upon MALDI, various carbohydrates [25] and posttranslationally modified peptides [26] form a fraction of labile molecular ions which lose characteristic fragments after acceleration. Compared to fully accelerated ions these fragment ions are defocused and give rise to signals exhibiting reduced resolution and shifted $\mathrm{m} / \mathrm{z}$ values in reflector MALDI mass spectra. These metastable ions are particularly abundant from carbohydrates carrying sialic acids and from peptides containing phosphorylated amino acids, carboxamidomethylated cysteines, or methionine sulfoxides.

Interestingly, fragment ions resulting from sidechain cleavages of DMA in peptides are observable in MALDI-TOF mass spectra too. A reflector MALDI mass spectrum recorded from a synthetic peptide containing four arginine residues, the sequence of which was derived from nucleolin, displayed only two very weak signals consistent with metastable losses of ammonia and carbodiimide (Figure 4a). In contrast, a MALDI mass spectrum acquired from the corresponding peptide with four aDMA residues shows a signal of medium intensity resulting from metastable loss of dimethylamine and a second signal attributable to two consecutive losses of this fragment (Figure $4 \mathrm{~b}$ ). In the present study, $\mathrm{m} / \mathrm{z}$ values of metastable ions were calculated by using an equation derived by Harvey et al. [25].

The reflector MALDI mass spectra of peptides GMMA-GLSLSR and G-sDMA-GLSLSR confirm that sDMA is considerably more susceptible to gas phase fragmentation in comparison to MMA (Figure 5). The MMA-containing peptide remains virtually intact, while the MALDI mass spectrum of the peptide with

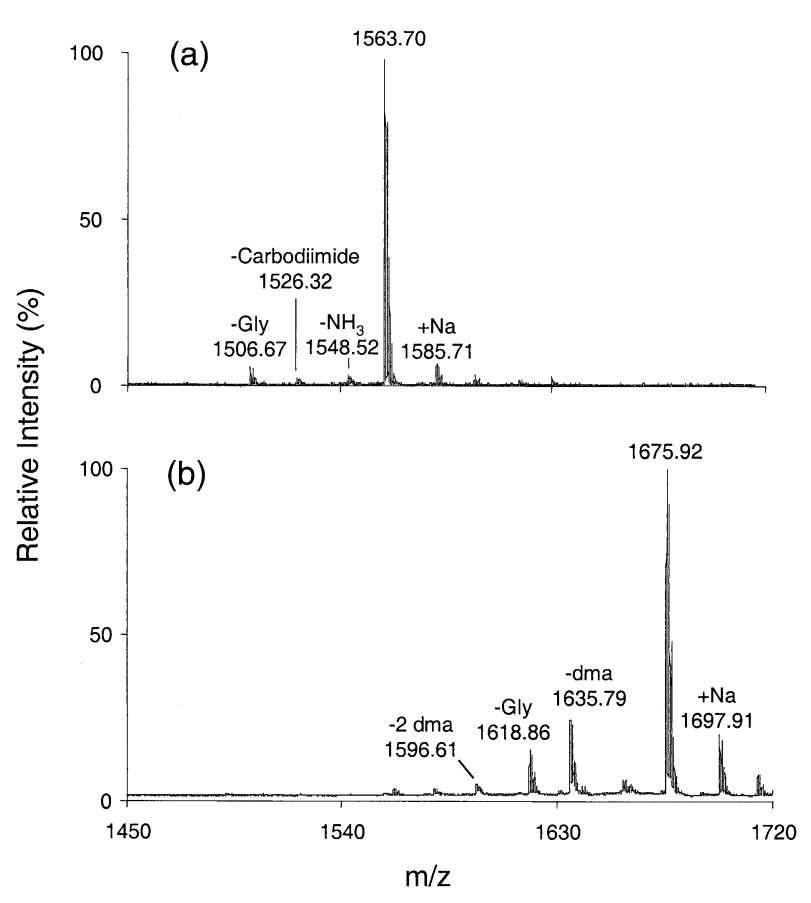

Figure 4. MALDI mass spectra recorded from 1 pmol (a) of peptide GRGGFGGRGGFRGGRGG- $\mathrm{NH}_{2}$ and (b) of peptide GaDMA-GGFGG-aDMA-GGF-aDMA-GG-aDMA-GG-NH ${ }_{2}$, which contains four aDMA residues.

sDMA displays two signals consistent with the release of methylamine and dimethylcarbodiimide from the sDMA side chain. These fragmentation patterns of the DMA side chains in standard MALDI mass spectra allow to unequivocally assign the isomeric form of the modified residues without the acquision of MALDI

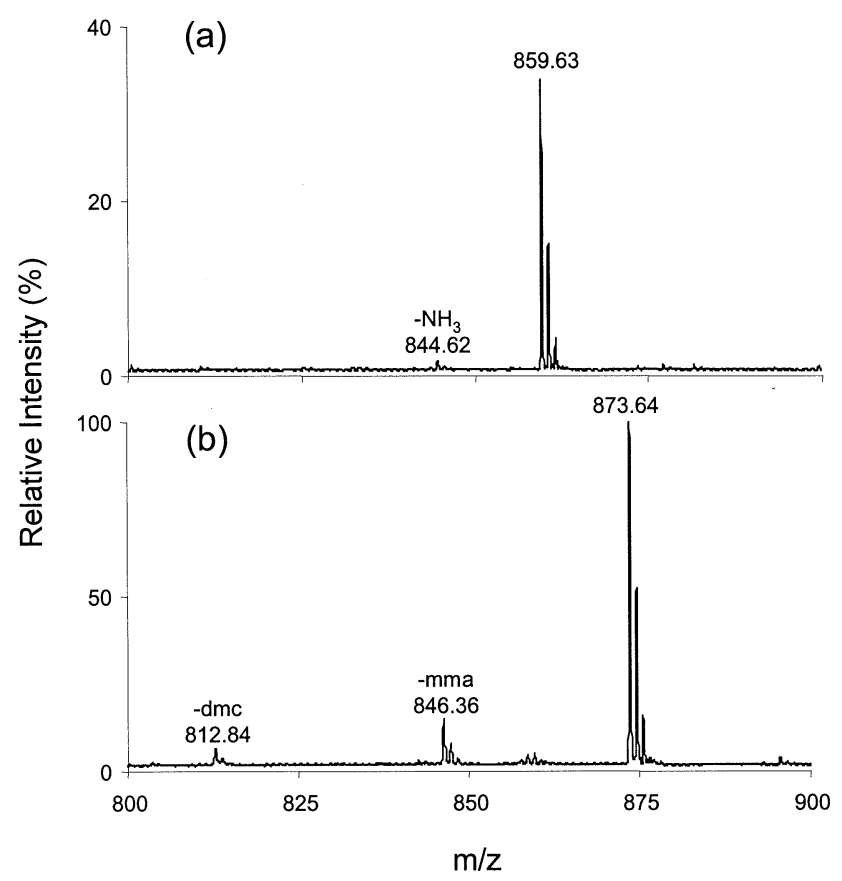

Figure 5. MALDI mass spectra (a) of peptide G-MMA-GLSLSR and $(\mathbf{b})$ of peptide G-sDMA-GLSLSR. 
post-source decay or CID spectra. Metastable ions resulting from the loss of dimethylamine can be used as evidence for the presence of aDMA in the corresponding peptide, while the loss of methylamine is a signature for sDMA.

Charged side chains of peptides such as the guanidino group of arginine tend to increase signal intensity in MALDI mass spectra [27]. For this reason, peptides containing modified arginines frequently give rise to relatively strong ion signals in MALDI spectra acquired from peptide mixtures, and the metastable fragment ion signals, which are less intense than the signals of intact DMA-containing peptides, are often well discernible even in spectra recorded from complex peptide mixtures. For example, human RNA-binding Ewing Sarcoma protein was shown, by using mass spectrometric peptide mass mapping and sequencing, to contain at least 29 dimethylated arginine residues [4]. We recorded MALDI mass spectra from trypsin and chymotrypsin digests of this protein and identified metastable peaks resulting from loss of dimethylamine, which indicated the presence of aDMA residues at 22 of these sites (unpublished data). Interestingly, a MALDI mass spectrum obtained from a trypsin digest of another methylated protein, SPT5, revealed the presence of both aDMA and sDMA in a single protein [28], which was confirmed by other methods.

Asymmetric dimethylation of the guanidinium group of arginine has several physicochemical effects: Increased hydrophobicity, a decrease of the hydrogen bonding ability, and a slightly lower pK value [29]. An additional effect of $\mathrm{N}^{\mathrm{G}}$-methylation is loss of planarity of the nitrogen atoms in the guanidinium group, probably due to steric hindrance. This might result in reduced delocalization energy of the positive charge and eventually in a decrease in the basicity and also in the stability of the guanidinium group. The loss of stability appears to be reflected in increased gas phase fragmentation of the guanidinium group. Both the ESI-MS/MS and the MALDI-TOF-MS data indicate that aDMA residues in peptides rather tend to fragment compared to monomethylated and unmodified arginines. Similar steric and stereoelectronic effects may facilitate the observed gas phase fragmentations of sDMA residues.

\section{Conclusions}

The ESI CID mass spectra recorded from peptides containing unmodified or $\mathrm{N}^{\mathrm{G}}$-methylated arginine residues display a variety of fragment ions resulting from cleavages of the guanidinium group. The ions include ammonium, carbodiimidium, and guanidinium ions as well as cyclic ions formed from arginine residues and peptide ions resulting from loss of neutral or charged fragments from the arginine side chain. ADMA and sDMA residues were found to be more prone to fragmentation than monomethylated and unmodified arginine. Loss of neutral or protonated dimethylamine and dimethylguanidine from aDMA and loss of neutral or protonated dimethylcarbodiimide and dimethylguanidine from sDMA are particularly predominant fragmentations. (Monomethylated) guanidine and guanidinium ions are also released from arginine and MMA residues. Precursor ion scanning measurements based on the selection of dimethylammonium as marker ion permit the specific detection of peptides with aDMA residues. A promising method for the analysis of this protein modification is the combination of precursor ion scanning with the acquisition of MS/MS spectra triggered by the detection of ion signals in the precursor ion scanning mode. Even MALDI-TOF mass spectra of peptides carrying aDMA exhibit metastable ion signals resulting from the loss of dimethylamine. Monomethylamine and dimethylcarbodiimide are released from sDMA, while monomethylarginine and unmodified arginine do not give rise to significant metastable ion signals in MALDI spectra.

\section{Acknowledgments}

The authors acknowledge the financial support by the Kanton of Zurich, Switzerland. They thank Dr. Larisa Belyanskaya and Professor Heinz Gehring (Institute of Biochemistry, University of Zurich) for providing EWS protein samples and Dr. Sergiy Chesnov (Institute of Biochemistry, University of Zurich) for quantitative amino acid analyses.

\section{References}

1. Paik, W. K.; Kim, S. Enzymatic Methylation of Protein Fractions from Calf Thymus Nuclei. Biochem. Biophys. Res. Commun. 1967, 29, 14-20.

2. Gary, J. D.; Clarke, S. RNA and Protein Interactions Modulated by Protein Arginine Methylation. Progr. Nucl. Acid Res. Mol. Biol. 1998, 61, 65-131.

3. McBride, A. E.; Silver, P. A. State of the Arg: Protein Methylation at Arginine Comes of Age. Cell 2001, 106, 5-8.

4. Belyanskaya, L. L.; Gehrig, P. M.; Gehring, H. Exposure on Cell Surface and Extensive Arginine Methylation of Ewing Sarcoma (EWS) Protein. J. Biol. Chem. 2001, 276, 18681-18687.

5. Baldwin, G. S.; Carnegie, P. R. Specific Enzymic Methylation of an Arginine in the Experimental Allergic Encephalomyelitis Protein from Human Myelin. Science 1971, 171, 579-581.

6. Brahms, H.; Meheus, L.; de Brabandere, V.; Fischer, U.; Lührmann, R. Symmetrical Dimethylation of Arginine Residues in Spliceosomal Sm Protein B/B' and the Sm-like Protein LSm4, and their Interaction with the SMN Protein. RNA 2001, 7, 1531-1542.

7. Frankel, A.; Yadav, N.; Lee, J.; Branscombe, T. L.; Clarke, S.; Bedford, M. T. J. The Novel Human Protein Arginine NMethyltransferase PRMT6 is a Nuclear Enzyme Displaying Unique Substrate Specificity. Biol. Chem. 2002, 277, 3537-3543.

8. Branscombe, T. L.; Frankel, A.; Lee, J.-H.; Cook, J. R.; Yang, Z.-H.; Pestka, S.; Clarke, S. PRMT5 (Janus Kinase-Binding Protein 1) Catalyzes the Formation of Symmetric Dimethylarginine Residues in Proteins. J. Biol. Chem. 2001, 276, 3297132976.

9. Zobel-Thropp, P.; Gary, J. D.; Clarke, S. $\delta$-N-Methylarginine is a Novel Posttranslational Modification of Arginine Residues in Yeast Proteins. J. Biol. Chem. 1998, 273, 29283-29286.

10. Székely, Z.; Zakhariev, S.; Guarnaccia, C.; Antcheva, N.; Pongor, S. A Highly Effective Method for Synthesis of $\mathrm{N}^{\omega_{-}}$ 
Substituted Arginines as Building Blocks for Boc/Fmoc Peptide Chemistry. Tetrahedron Lett. 1999, 40, 4439-4442.

11. Lischwe, M. A.; Roberts, K. D.; Yeoman, L. C.; Busch, H. J. Nucleolar Specific Acidic Phosphoprotein C23 is Highly Methylated. Biol. Chem. 1982, 257, 14600-14602.

12. Huddlestone, M. J.; Bean, M. F.; Carr, S. A. Collisional Fragmentation of Glycopeptides by Electrospray Ionization LC/MS and LC/MS/MS: Methods for Selective Detection of Glycopeptides in Protein Digests. Anal. Chem. 1993, 65, 877884.

13. Wilm, M.; Neubauer, G.; Mann, M. Parent Ion Scans of Unseparated Peptide Mixtures. Anal. Chem. 1996, 68, 527-533.

14. Rappsilber, J.; Friesen, W. J.; Paushkin, S.; Dreyfuss, G.; Mann, M. Detection of Arginine Dimethylated Peptides by Parallel Precursor Ion Scanning Mass Spectrometry in Positive Ion Mode. Anal. Chem. 2003, 75, 3107-3114.

15. Johnson, R. S.; Biemann, K. Computer Program (SEQPEP) to Aid in the Interpretation of High-energy Collision Tandem Mass Spectra of Peptides. Biomed. Environ. Mass Spectrom. 1989, 18, 945-957.

16. Falick, A. M.; Hines, W. M.; Medzihradszky, K. F.; Baldwin, M. A.; Gibson, B. W. Low Mass Ions Produced from Peptides by High-Energy Collision-Induced Dissociation in Tandem Mass Spectrometry. J. Am. Soc. Mass Spectrom. 1993, 4, 882-893.

17. Papayannopoulos, I. A. The Interpretation of Collision-Induced Dissociation Tandem Mass Spectra of Peptides. Mass Spectrom. Rev. 1995, 14, 49-73.

18. Fields, G. B.; Noble, R. L. Solid Phase Peptide Synthesis Utilizing 9-Fluorenylmethoxycarbonyl Amino Acids. Int. J. Peptide Protein Res. 1990, 35, 161-214.

19. Yuan, M.; Namikoshi, M.; Otsuki, A.; Rinehart, K. L.; Sivonen, K.; Watanabe, M. F. Low-Energy Collisionally Activated Decomposition and Structural Characterization of Cyclic Heptapeptide Microcystins by Electrospray Ionization Mass Spectrometry. J. Mass Spectrom. 1999, 34, 33-43.

20. Yagüe, J.; Vázquez, J.; López de Castro, J. A. A Post-Translational Modification of Nuclear Proteins, $N^{G}, N^{G}$-Dimethyl-Arg Found in a Natural HLA Class I Peptide Ligand. Protein Sci. 2000, 9, 2210-2217.
21. Vishwanathan, K.; Tackett, R. L.; Stewart, J. T.; Bartlett, M. G. Determination of Arginine and Methylated Arginines in Human Plasma by Liquid Chromatography-Tandem Mass Spectrometry. J. Chromatogr. B 2000, 748, 157-166.

22. Carr, S. A.; Huddlestone, M. J.; Annan, R. S. Selective Detection and Sequencing of Phosphopeptides at the Femtomole Level by Mass Spectrometry. Anal. Biochem. 1996, 239, 180-192.

23. Steen, H.; Küster, B.; Fernandez, M.; Pandey, A.; Mann, M. Detection of Tyrosine Phosphorylated Peptides by Precursor Ion Scanning Quadrupole Mass Spectrometry in Positive Ion Mode. Anal. Chem. 2001, 73, 1440-1448.

24. Haynes, P. A.; Aebersold, R. Simultaneous Detection and Identification of O-GlcNAc-Modified Glycoproteins Using Liquid Chromatography-Tandem Mass Spectrometry. Anal. Chem. 2000, 72, 5402-5410.

25. Harvey, D. J.; Hunter, A. P.; Bateman, R. H.; Brown, J.; Critchley, G. Relationship Between In-Source and Post-Source Fragment Ions in the Matrix-Assisted Laser Desorption (Ionization) Mass Spectra of Carbohydrates Recorded with Reflectron Time-of-Flight Mass Spectrometers. Int. J. Mass Spectrom. 1999, 188, 131-146.

26. Wirth, U.; Müller, D.; Schindler, P.; Lange, J.; van Oostrum, J. Post-Translational Modification Detection Using Metastable Ions in Reflector Matrix-Assisted Laser Desorption/Ionization-Time of Flight Mass Spectrometry. Proteomics 2002, 2, 1445-1451.

27. Krause, E.; Wenschuh, H.; Jungblut, P. R. The Dominance of Arginine-Containing Peptides in MALDI-Derived Tryptic Mass Fingerprints of Proteins. Anal. Chem. 1999, 71, 4160-4165.

28. Kwak, Y. T.; Guo, J.; Prajapati, S.; Park, K.-J.; Surabhi, R. M.; Miller, B.; Gehrig, P.; Gaynor, R. B. Methylation of SPT5 Regulates its Interaction with RNA Polymerase II and Transcriptional Elongation Properties. Mol. Cell 2003, 11, 10551066.

29. Raman, B.; Guarnaccia, C.; Nadassy, K.; Zakhariev, S.; Pintar, A.; Zanuttin, F.; Frigyes, D.; Acatrinei, C.; Vindigni, A.; Pongor, G.; Pongor, S. N ${ }^{\omega}$-Arginine Dimethylation Modulates the Interaction between a Gly/Arg-Rich Peptide from Human Nucleolin and Nucleic Acids. Nucleic Acids Res. 2001, 29, 3377-3384. 